\title{
Condição bucal dos pacientes admitidos em Unidades de Terapia Intensiva
}

\author{
Oral condition of patients admitted in Intensive Care Units \\ Estado bucal de pacientes ingresados en Unidades de Cuidados Intensivos
}

Recebido: 30/03/2021 | Revisado: 09/04/2021 | Aceito: 13/04/2021 | Publicado: 24/04/2021

\author{
Hugo Angelo Gomes de Oliveira \\ ORCID: https://orcid.org/0000-0001-5176-034X \\ Real Hospital Português de Beneficência em Pernambuco, Brasil \\ E-mail: hugoangeloo@gmail.com \\ Marcelo Vieira da Costa Almeida \\ ORCID: https://orcid.org/0000-0002-8521-423X \\ Real Hospital Português de Beneficência em Pernambuco, Brasil \\ E-mail: marcelo-vca@ hotmail.com \\ Luciana Ferraz Gominho \\ ORCID: https://orcid.org/0000-0002-7881-0316 \\ Universidade Federal da Paraíba, Brasil \\ E-mail: fggf.end@gmail.com \\ Maria Kaline Romeiro Teodoro \\ ORCID: https://orcid.org/0000-0002-6482-0628 \\ Real Hospital Português de Beneficência em Pernambuco, Brasil \\ E-mail: kaline_rote@hotmail.com \\ Lucia de Fátima Cavalcanti dos Santos \\ ORCID: https://orcid.org/0000-0002-7107-1901 \\ Real Hospital Português de Beneficência em Pernambuco, Brasil \\ E-mail: luciasantoslaser7@gmail.com \\ Antonio Carlos Moura de Albuquerque Melo \\ ORCID: https://orcid.org/0000-0002-9336-7797 \\ Real Hospital Português de Beneficência em Pernambuco, Brasil \\ E-mail: carlosmouramelo@gmail.com \\ Ully Dias Nascimento Távora Cavalcanti \\ ORCID: https://orcid.org/0000-0003-4748-1692 \\ Real Hospital Português de Beneficência em Pernambuco, Brasil \\ E-mail: ullydias@gmail.com
}

\begin{abstract}
Resumo
Objetivo: Identificar a condição bucal dos pacientes admitidos em unidades de terapia intensiva. Metodologia: Tratase de um estudo observacional retrospectivo utilizando dados secundários de prontuário de pacientes admitidos nas unidades de terapia intensiva de um hospital de beneficência de alta complexidade da cidade do Recife, atendidos pela equipe de Odontologia Hospitalar no período de março de 2019 a fevereiro de 2020. Resultados: A amostra envolveu um total de 1831 pacientes, com idade média de 67,63 anos, sendo 50,8\% do sexo masculino. As doenças neurológicas $(19,9 \%)$ e do aparelho respiratório $(18,8 \%)$ foram as responsáveis pelos maiores índices de admissões. As principais alterações bucais evidenciadas foram: cálculo dental $(27,2 \%)$, fratura dentária $(17,1 \%)$, lesão/infecção $(14,4 \%)$ e ressecamento labial/bucal (10\%). Conclusão: Conclui-se que o cálculo dental foi a alteração bucal mais frequente nos pacientes avaliados, observado com maior número nos pacientes com doenças neurológicas e do aparelho respiratório. A presença de infecção/lesão na cavidade bucal foi diagnóstica em maior índice nos pacientes oncológicos.
\end{abstract}

Palavras-chave: Diagnóstico bucal; Saúde bucal; Higiene bucal; Controle de infecç̃os; Unidades de terapia intensiva.

\begin{abstract}
Objective: To identify the oral condition of patients admitted to intensive care units. Methodology: This is a retrospective observational study using secondary data from medical records of patients admitted to the intensive care units of a high complexity charitable hospital in the city of Recife, attended by the Hospital Dentistry team from March 2019 to February 2020. Results: The sample involved a total of 1831 patients, with an average age of 67.63 years, $50.8 \%$ of whom were male. Neurological $(19.9 \%)$ and respiratory $(18.8 \%)$ diseases were responsible for the highest admission rates. The main oral alterations evidenced were: tartar $(27.2 \%)$, dental fracture $(17.1 \%)$, injury / infection (14.4\%) and dry lip / mouth (10\%). Conclusion: It is concluded that tartar was the most frequent oral alteration in the evaluated patients, observed with greater number in patients with neurological and respiratory diseases. The presence of infection / injury in the oral cavity was diagnosed at a higher rate in cancer patients.
\end{abstract}

Keywords: Oral diagnosis; Oral health; Oral hygiene; Infection control; Intensive care units. 


\section{Resumen}

Objetivo: Identificar la condición bucal de los pacientes ingresados en unidades de cuidados intensivos. Metodología: Se trata de un estudio observacional retrospectivo que utiliza datos secundarios de historias clínicas de pacientes ingresados en las unidades de cuidados intensivos de un hospital benéfico de alta complejidad en la ciudad de Recife, atendido por el equipo de Odontología del Hospital de marzo de 2019 a febrero de 2020. Resultados: La La muestra involucró a un total de 1.831 pacientes, con una edad promedio de 67,63 años, de los cuales el 50,8\% eran varones. Las enfermedades neurológicas $(19,9 \%)$ y las respiratorias $(18,8 \%)$ fueron las responsables de las mayores tasas de ingresos. Las principales alteraciones bucales evidenciadas fueron: sarro (27,2\%), fractura dentaria (17,1\%), lesión / infección $(14,4 \%)$ y sequedad labial / bucal (10\%). Conclusión: Se concluye que el sarro fue la alteración bucal más frecuente en los pacientes evaluados, observándose con mayor número en pacientes con enfermedades neurológicas y respiratorias. La presencia de infección / lesión en la cavidad oral se diagnosticó con mayor frecuencia en pacientes con cáncer.

Palabras clave: Diagnóstico oral; Salud bucal; Higiene bucal; Control de infección; Unidades de cuidados intensivos.

\section{Introdução}

Atualmente, as infecções hospitalares são uma das principais causas de mortalidade e morbidade dos pacientes internados (Meidani, Khorvash, Abbasi, Cheshmavar, \& Tavakoli, 2018). Considerações importantes têm sido relatadas em estudos onde apontam o biofilme bucal como destaque para o desenvolvimento de infecções bacterianas, virais e/ou fúngicas, especialmente em pacientes internados em unidade de terapia intensiva (UTI) (Vidal, 2014). Tal relação é atribuída à desordem homeostática microbiana bucal (Tulio et al., 2018).

A qualidade do cuidado bucal está relacionada ao número de bactérias presentes na boca. Geralmente, pacientes graves sob ventilação mecânica (VM) invasiva cursam com péssimas condições de higiene bucal, além disso, esses indivíduos são expostos a inúmeros outros fatores como redução do mecanismo natural de limpeza da cavidade bucal por meio de movimentos da língua e fluxo de saliva reduzido, todos contribuintes para a formação do biofilme e colonização por patógenos respiratórios (Munro \& Grap, 2004). Quando comparados com aqueles que não estão internados e com adequada higiene bucal, observa-se precárias condições de higiene bucal nos pacientes internados, por si só, se relacionam com maior ocorrência de infecções respiratórias, febre e pneumonia (Scannapieco, 2006).

Conforme ocorre uma deficiência da higienização bucal dos pacientes internados, a cavidade bucal, que é colonizada por uma grande variedade de microrganismos, torna-se um reservatório de microrganismos patogênicos capazes de ocasionar infecções, tanto na cavidade bucal, quanto de maneira sistêmica (Disner, Freddo, \& Lucietto, 2018; Emery \& Guido-Sanz, 2019).

Tem sido relatado que a saúde bucal é de suma importância em pacientes em VM na UTI e esses cuidados são de difícil realização por outros profissionais não capacitados. Assim, é importante determinar o impacto dos protocolos de cuidados bucais na saúde dos pacientes que estão nesse ambiente (Blum, Da Silva, Baeder, \& Bona, 2018).

Diante do exposto, o objetivo deste trabalho foi identificar nos registros a condição bucal dos pacientes admitidos em unidades de terapia intensiva atendidos pela equipe de Odontologia Hospitalar responsável pelo hospital cenário do estudo.

\section{Metodologia}

Este estudo foi aprovado pelo Comitê de Ética em Pesquisa do Real Hospital Português de Beneficência em Pernambuco (RHP) sob o número do parecer 4.315.188. Por se tratar de um estudo observacional retrospectivo, utilizando dados secundários de prontuário, não foi necessário obter o Termo de Consentimento Livre e Esclarecido (TCLE).

O estudo foi realizado no RHP, considerado como de alta complexidade, situado na cidade do Recife - PE - Brasil. A coleta foi realizada através de registros de prontuários de quatro UTIs geral contendo 68 leitos no total no universo de 192 leitos. A amostra foi constituída por indivíduos adultos admitidos nas UTIs, atendidos pela equipe de Odontologia Hospitalar, com idade igual ou superior a 18 anos, no período de março de 2019 a fevereiro de 2020. 
Para a coleta de dados dos pacientes foi considerada: número total de dentes, presença/ausência de prótese dentária, cálculo dental, fratura dentária, mobilidade dentária, ressecamento da mucosa bucal e labial, presença de lesão/infecção intrabucal, infecção fúngica, sangramento gengival, supuração intrabucal, mucosite oral (MO) e o grau associado da mucosite.

Os dados foram tabulados em planilha de Excel e transferidos posteriormente para o software estatístico SPSS (Statistical Package for Social Sciences) ver. 21.0. Com o objetivo de caracterizar a amostra estudada, foram calculadas as frequências relativas (percentuais) e absolutas (N) das classes de cada variável.

\section{Resultados e Discussão}

Este estudo incluiu 1831 prontuários de pacientes admitidos nas UTIs.

A Tabela 1 apresenta, em valores absolutos e relativos, os dados gerais da amostra.

Tabela 1 - Dados gerais da amostra.

\begin{tabular}{|c|c|c|}
\hline Dados gerais & 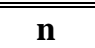 & $\%$ \\
\hline \multicolumn{3}{|l|}{ Sexo } \\
\hline Masculino & 930 & 50,8 \\
\hline Feminino & 901 & 49,2 \\
\hline Total & 1831 & 100 \\
\hline Média de idade & 67,6 & - \\
\hline \multicolumn{3}{|l|}{ Motivo da admissão } \\
\hline Doenças neurológicas & 364 & 19,9 \\
\hline Doenças do aparelho respiratório & 345 & 18,8 \\
\hline Doenças oncológicas & 240 & 13,1 \\
\hline Doenças cardiovasculares & 210 & 11,5 \\
\hline Doenças renais & 182 & 9,9 \\
\hline Doenças do aparelho gastrointestinal & 134 & 7,3 \\
\hline Sepse & 94 & 5,1 \\
\hline Doenças hepáticas & 65 & 3,5 \\
\hline Doenças psiquiátricas & 46 & 2,5 \\
\hline Acidentes & 33 & 1,8 \\
\hline Doenças metabólicas & 25 & 1,4 \\
\hline Pós-operatório & 21 & 1,1 \\
\hline Doenças virais & 18 & 1,0 \\
\hline Doenças degenerativas do sistema nervoso & 16 & 0,9 \\
\hline Doenças sanguíneas & 12 & 0,7 \\
\hline Pré-eclâmpsia & 10 & 0,5 \\
\hline Doenças autoimune & 9 & 0,5 \\
\hline Doenças óssea & 5 & 0,3 \\
\hline Choque anafilático & 2 & 0,1 \\
\hline Total & 1831 & 100 \\
\hline
\end{tabular}

Fonte: Autores.

Em relação ao número total de dentes presentes, foi observado que os pacientes possuíam em média 16,07 dentes presentes na boca no momento do atendimento.

O Gráfico 1 apresenta, em valores relativos, os dados da condição bucal dos pacientes avaliados. 
Research, Society and Development, v. 10, n. 4, e58910414444, 2021

(CC BY 4.0) | ISSN 2525-3409 | DOI: http://dx.doi.org/10.33448/rsd-v10i4.14444

Gráfico 1 - Condição bucal dos pacientes avaliados.

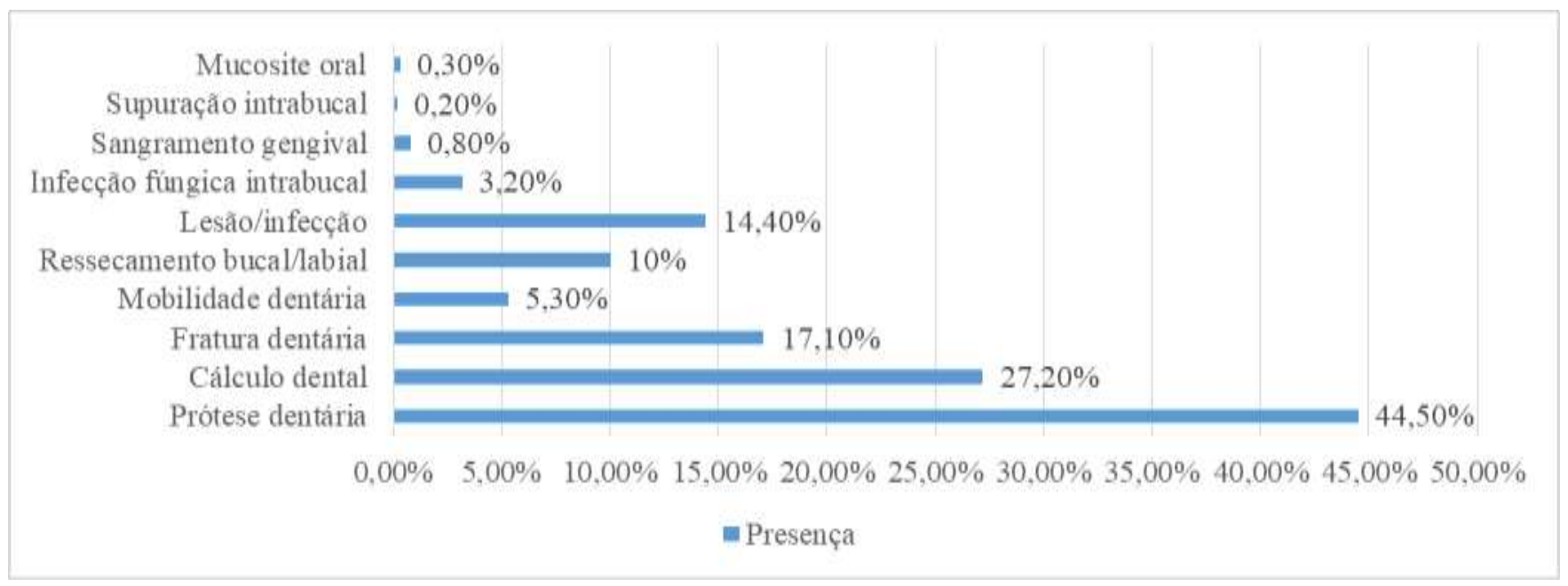

Fonte: Autores.

O Gráfico 2 apresenta, em valores relativos, os aspectos periodontais (cálculo dental e mobilidade dentária) dos pacientes avaliados.

Gráfico 2 - Presença de cálculo dental e mobilidade dentária conforme o Código Internacional de Doenças (CID).

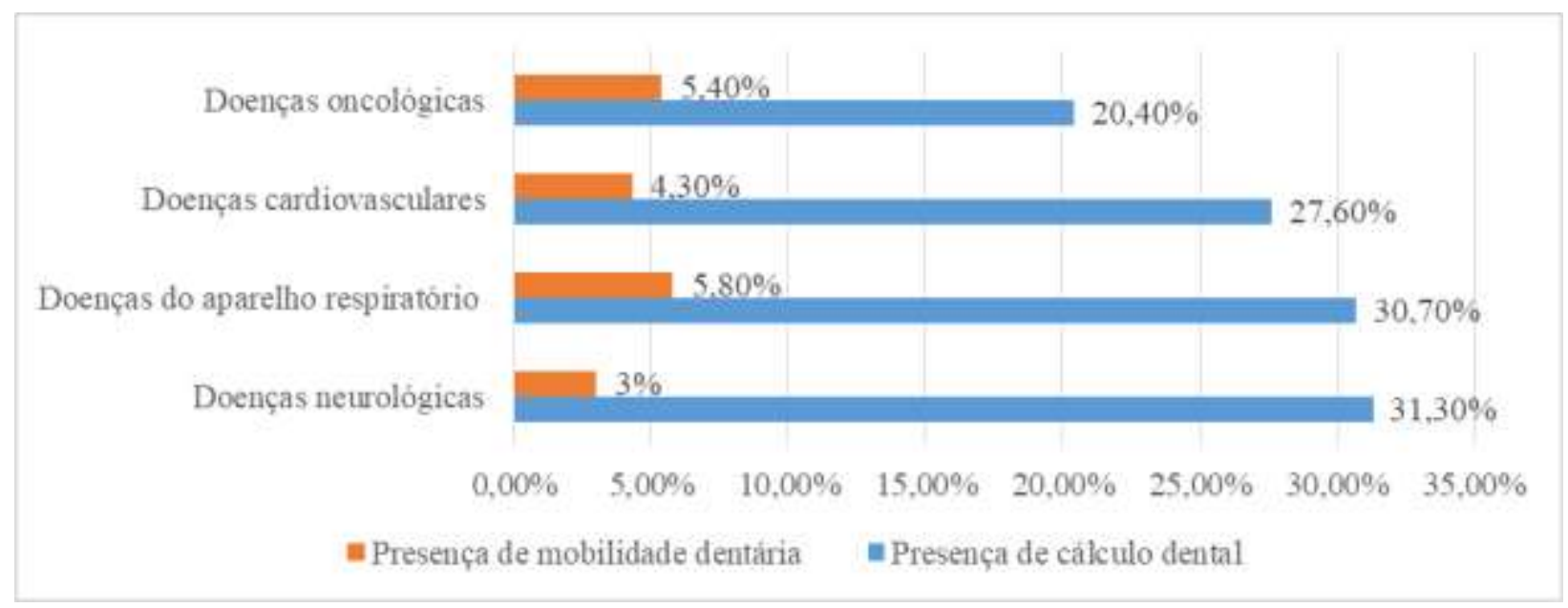

Fonte: Autores.

O Gráfico 3 apresenta, em valores relativos, a presença de infecção ou lesão intrabucal dos pacientes avaliados. 
Research, Society and Development, v. 10, n. 4, e58910414444, 2021

(CC BY 4.0) | ISSN 2525-3409 | DOI: http://dx.doi.org/10.33448/rsd-v10i4.14444

Gráfico 3 - Presença de infecção ou lesão intrabucal conforme o CID.

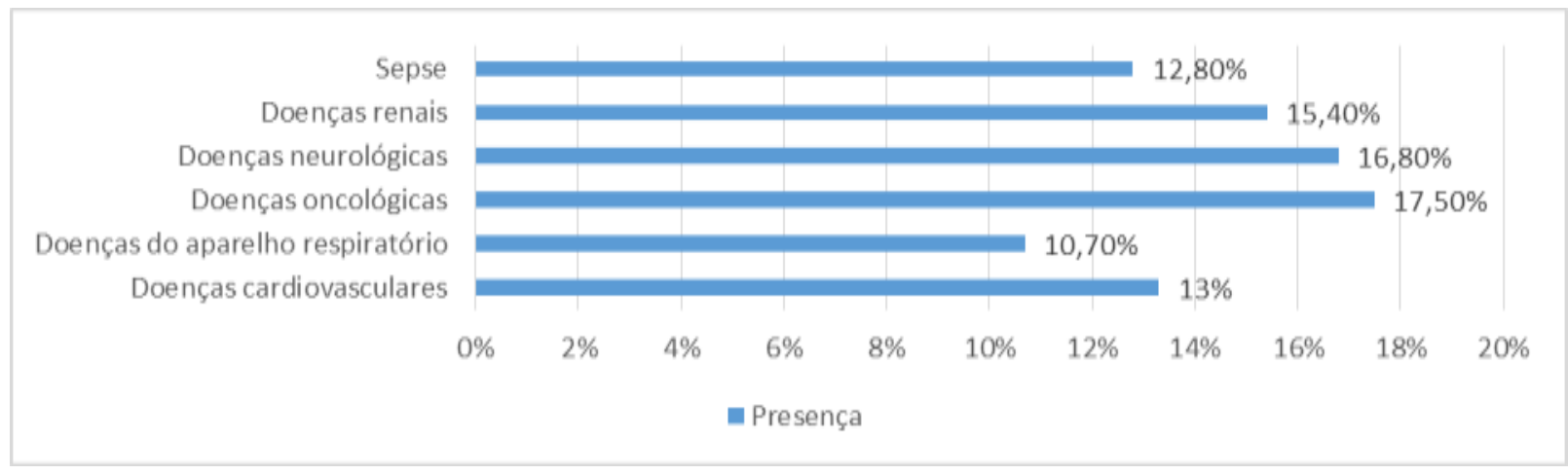

Fonte: Autores.

O Gráfico 4 apresenta, em valores relativos, a presença de fratura dentária dos pacientes avaliados.

Gráfico 4 - Presença de fratura dentária conforme o CID.

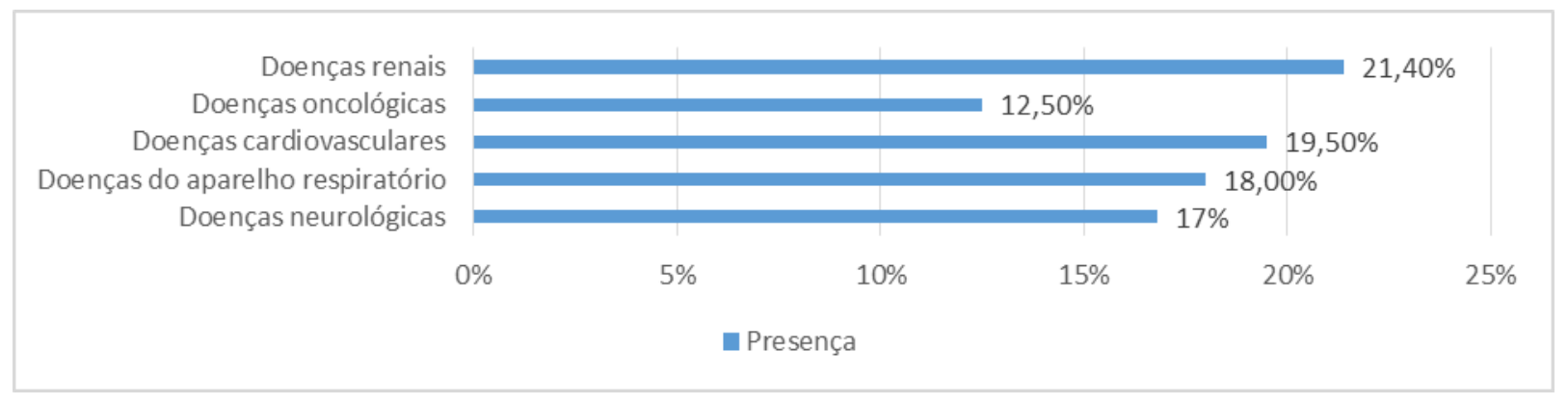

Fonte: Autores.

O Gráfico 5 apresenta, em valores relativos, a presença de ressecamento bucal/labial dos pacientes avaliados.

Gráfico 5 - Presença de ressecamento bucal/labial conforme o CID.

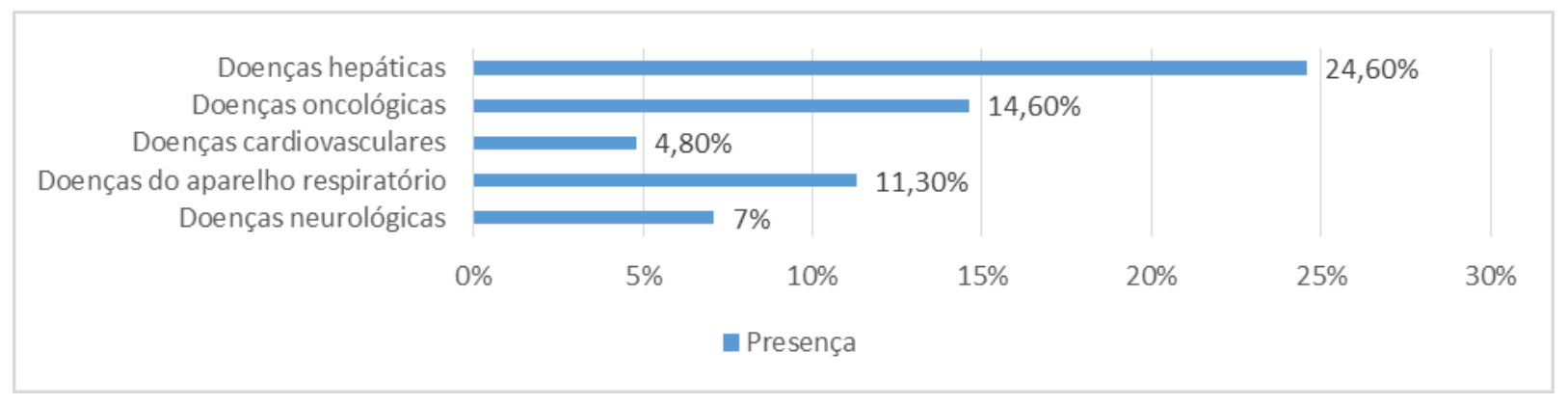

Fonte: Autores.

Foi observado que 6 pacientes $(0,3 \%)$ foram diagnosticados com MO, sendo três pacientes com MO grau I (50\%), dois pacientes com grau II $(33,3 \%)$ e apenas um com grau III $(16,6 \%)$, observado mais em pacientes com câncer de mama $(33,3 \%)$.

O Gráfico 6 apresenta, em valores relativos, a presença de lesão fúngica intrabucal nos pacientes avaliados. 
Gráfico 6 - Presença de lesão fúngica intrabucal conforme o CID.

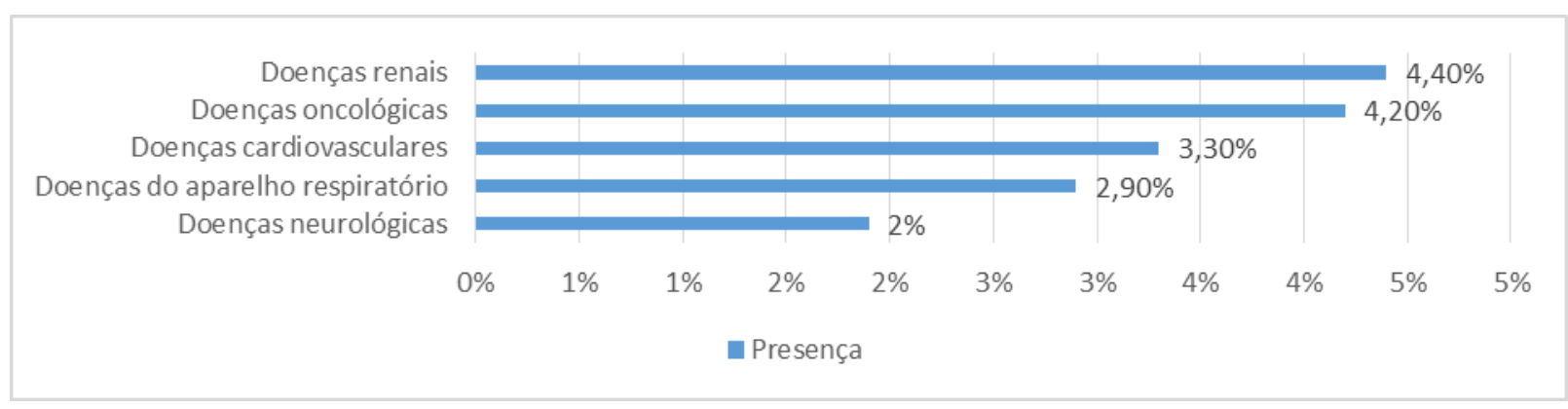

Fonte: Autores.

A demanda por terapia intensiva geralmente excede a disponibilidade de tal tratamento (Sprung et al., 2013). Populações envelhecidas e mudanças nos padrões de doenças levaram a um aumento na demanda por serviços de terapia intensiva em muitos países. O tratamento em UTI é geralmente considerado caro e a capacidade precisa aumentar substancialmente nas próximas décadas para que os sistemas de saúde atendam às crescentes demandas (Angus et al., 2004; Laake et al., 2010).

Em um estudo norurguês utilizando uma amostra de 30.712 admissões em UTI, no qual constataram uma média de idade de 62.7 (Lindemark, Haaland, Kvåle, Flaatten, \& Johansson, 2015). Já no Brasil, foi visto em 2016, uma prevalência de 42.23\% de pacientes do sexo masculino e 57.77\% do sexo feminino (da Silva, El Kadre, Kudo, Junior, \& Saraiva, 2016), número próximo ao encontrado neste estudo.

Divergindo com os achados no presente estudo, uma pesquisa realizada nos Estados Unidos da América (EUA), avaliando 102.346 admissões, e no Reino Unido, avaliando 70.439 admissões, no qual constataram que nos EUA e no Reino Unido, o maior motivo para a internação dos pacientes foi por doenças cardíacas sendo 44,6\% e 27,1\% respectivamente (Wunsch, Angus, Harrison, Linde-Zwirble, \& Rowan, 2011).

A presença de um profissional da odontologia ajuda a manter a adesão aos protocolos de saúde bucal, além de apoiar e dar assistência à equipe para enfrentar as eventuais dificuldades durante os cuidados ao paciente. É também importante salientar a associação entre treinamento adequado da equipe e a presença de um profissional em odontologia na rotina da UTI (Gmür, Irani, Attin, Menghini, \& Schmidlin, 2013; Jordan et al., 2014).

Em um estudo realizado entre dezembro de 2015 e junho de 2016, com 202 pacientes em 20 unidades de terapia intensiva de 6 hospitais da Turquia, observou-se que os pacientes avaliados tinham uma perda dentária de 6,6 em média por pessoa, número muito abaixo do que o encontrado na presente pesquisa (16,07 dentes) (Celik \& Eser, 2017).

Dentre as doenças sistêmicas, as que acumulam mais evidências científicas da sua relação com as doenças periodontais, são as doenças respiratórias, além disso, as doenças cardiovasculares e o diabetes mellitus (DM) (Ahmed \& Tanwir, 2015; Liccardo et al., 2019; Macedo, Saba-Chujfi, Pereira, Costa, \& Melo Neto, 2010; Sanz et al., 2020; Scannapieco, 2006). As periodontopatias podem influenciar o curso das infecções respiratórias destacando-se as pneumonias, sendo doenças do aparelho respiratório responsáveis pelo segundo maior índice de admissões nas UTIs no presente estudo (18,8\%) (Scannapieco \& Rethman, 2003). Ao avaliar os aspectos periodontais, foi observado que 27,2\% dos pacientes possuíam acúmulo de cálculo dental nos dentes, 5,3\% dos pacientes tinham algum grau de mobilidade dentária e apenas $0,8 \%$ dos pacientes apresentavam sangramento gengival visível ao exame intrabucal. Em um estudo realizado avaliando clinicamente a cavidade bucal de 35 pacientes, foi constatado que todos os pacientes apresentavam biofilme bucal na admissão em UTI, sendo que 20 pacientes $(57 \%)$ apresentavam biofilme por meio de simples visão, em 13 pacientes (37\%) havia muita placa, inclusive nos espaços interdentais, podendo haver cálculos, e em dois pacientes o biofilme era detectado ao se passar a sonda periodontal 
(Da Cruz, De Morais, \& Trevisani, 2014).

A Proteína C Reativa (PCR), presente nas doenças periodontais, é um marcador de processos inflamatórios liberada pelo fígado, indivíduos com PCR cronicamente elevada apresentam risco significativamente aumentado de desenvolver eventos cardiovasculares sendo assim, uma ferramenta de diagnóstico, aumentando a conscientização sobre o papel da inflamação de todas as fontes, inclusive na bucal (Ridker, Buring, Shih, Matias, \& Hennekens, 1998). Um estudo com 9.760 adultos dos EUA em 1993, descobriu que pessoas com periodontite tinham um risco aumentado de $25 \%$ de doença cardíaca em relação àqueles com doença periodontal mínima (DeStefano, Anda, Kahn, Williamson, \& Russell, 1993).

Um hospital universitário brasileiro realizou um estudo com 188 pacientes internados, sendo observado lesões de origem traumática na mucosa bucal foram relatadas em 16,49\% dos prontuários (31 pacientes), destes pacientes, 11 apresentavam lesões $(35,48 \%)$ localizadas na língua, 15 (48,39\%) lesões em mucosa jugal ou labial e 12 (38,71\%) lesões em gengiva ou rebordo gengival além de 10 casos $(5,32 \%)$ de hematomas por trauma e 38 pacientes $(20,21 \%)$ apresentaram candidíase (Costa, Saldanha, Sousa, \& Gaetti-Jardim, 2016).

Foi observado que 183 pacientes (10\%) apresentavam ressecamento bucal/labial, observado em 14,6\% dos pacientes oncológicos, resultado próximo encontrado em um estudo realizado por Silva (2019) ao avaliar a condição bucal de 70 pacientes internados em UTI, no qual observou a presença de ressecamento bucal/labial em 65,7\% dos pacientes avaliados (Silva, 2019).

Contra premissas intuitivas, os principais motivos para a admissão de pacientes com câncer na UTI são hipotensão (choque) e insuficiência respiratória aguda, de natureza não infecciosa. Estes são seguidos por sepse, lesão renal aguda, sangramento, emergências oncológicas e cuidados pós-operatórios após ressecção de tumores complexos ou grandes. O câncer brônquico é o tipo de tumor sólido mais comum em adultos encontrados na UTI; leucemia e linfoma são os cânceres hematológicos mais comuns (Kostakou, Rovina, Kyriakopoulou, Koulouris, \& Koutsoukou, 2014). Em combinação com o presente estudo, onde foi observado que o câncer foi responsável por 240 admissões $(13,1 \%)$ dos pacientes avaliados na UTI.

A MO induzida por quimioterapia e radioterapia é uma carga significativa para pacientes com câncer. Os sintomas da MO após a quimioterapia podem progredir de eritema, rachaduras e inflamação para dor, sangramento e ulceração (Epstein \& Schubert, 1999; Raber-Durlacher et al., 2000). No presente estudo foi observado que apenas $0,6 \%$ (n=6) dos pacientes foram admitidos com MO, corroborando com os achados em um estudo que avaliou o perfil bucal de 70 pacientes internados em UTI no qual foi constatado que apenas $5(7,1 \%)$ pacientes apresentaram lesões de MO (Silva, 2019).

\section{Conclusão}

Diante dos resultados encontrados, conclui-se que as doenças neurológicas e do aparelho respiratório foram as responsáveis pelo maior índice de admissões na UTI. O cálculo dental foi a alteração bucal mais frequente nos pacientes avaliados, observado com maior número nos pacientes com doenças neurológicas e do aparelho respiratório.

Espera-se que os resultados obtidos com este estudo possam reforçar a importância da atuação do Cirurgião-Dentista em pacientes internados em UTIs, visando orientar o planejamento das ações em saúde bucal e aperfeiçoar o atendimento odontológico oferecido no ambiente hospitalar.

A partir desta pesquisa a demanda pelo serviço odontológico pode-se quantificar e assim justificar a necessidade de implantação do serviço de odontologia hospitalar aos pacientes internados em UTIs. Portanto, torna-se imprescindível a atuação do Cirurgião-Dentista em UTI, visando a prevenção e tratamento de possíveis alterações que possam contribuir com o maior tempo de internamento e maior morbidade para o paciente, consequente com o aumento dos custos hospitalares. 


\section{Referências}

Ahmed, U., \& Tanwir, F. (2015). Association of periodontal pathogenesis and cardiovascular diseases: A literature review. Oral Health and Preventive Dentistry, 13(1), 21-27.

Angus, D. C., Barnato, A. E., Linde-Zwirble, W. T., Weissfeld, L. A., Watson, R. S., Rickert, T., \& Rubenfeld, G. D. (2004). Use of intensive care at the end of life in the United States: An epidemiologic study. Critical Care Medicine, 32(3), 638-643.

Blum, D. F. C., Da Silva, J. A. S., Baeder, F. M., \& Bona, A. D. (2018). The practice of dentistry in intensive care units in Brazil. Revista Brasileira de Terapia Intensiva, 30(3), 327-332.

Celik, G. G., \& Eser, I. (2017). Examination of intensive care unit patients' oral health. International Journal of Nursing Practice, $23(6)$, 1-9.

Costa, D. C. da, Saldanha, K. F. D., Sousa, A. S. de, \& Gaetti-Jardim, E. C. (2016). Perfil de saúde bucal dos pacientes internados no Hospital Universitário Maria Aparecida Pedrossian, Campo Grande (MS). Archives of Health Investigation, 5(2), 70-77.

Da Cruz, M. K., De Morais, T. M. N., \& Trevisani, D. M. (2014). Clinical assessment of the oral cavity of patients hospitalized in an intensive care unit of an emergency hospital. Revista Brasileira de Terapia Intensiva, 26(4), 379-383.

DeStefano, F., Anda, R. F., Kahn, H. S., Williamson, D. F., \& Russell, C. M. (1993). Dental disease and risk of coronary heart disease and mortality. British Medical Journal, 306(6879), 688-691.

Disner, O., Freddo, S. L., \& Lucietto, D. A. (2018). Oral Health in Intensive Care Units: Level of Information, Practices and Demands of Health Professionals. Journal of Health Sciences, 20(4), 252.

Emery, K. P., \& Guido-Sanz, F. (2019). Oral care practices in non-mechanically ventilated intensive care unit patients: An integrative review. In Journal of Clinical Nursing. 28(13-14), 2462-2471.

Epstein, J. B., \& Schubert, M. M. (1999). Oral mucositis in myelosuppressive cancer therapy. Oral Surgery, Oral Medicine, Oral Pathology, Oral Radiology, and Endodontics, 88(3), 273-276.

Gmür, C., Irani, S., Attin, T., Menghini, G., \& Schmidlin, P. R. (2013). Survey on Oral Hygiene Measures for Intubated Patients in Swiss Intensive Care Units. 123, 394-409.

Jordan, A., Badovinac, A., Špalj, S., Par, M., Šlaj, M., \& Plančak, D. (2014). Factors influencing intensive care nurses’ knowledge and attitudes regarding ventilator-associated pneumonia and oral care practice in intubated patients in Croatia. American Journal of Infection Control, $42(10), 1115-1117$.

Kostakou, E., Rovina, N., Kyriakopoulou, M., Koulouris, N. G., \& Koutsoukou, A. (2014). Critically ill cancer patient in intensive care unit: Issues that arise. Journal of Critical Care, 29(5), 817-822.

Laake, J. H., Dybwik, K., Flaatten, H. K., Fonneland, I. L., Kvale, R., \& Strand, K. (2010). Impact of the post-World War II generation on intensive care needs in Norway. Acta Anaesthesiologica Scandinavica, 54(4), 479-484.

Liccardo, D., Cannavo, A., Spagnuolo, G., Ferrara, N., Cittadini, A., Rengo, C., \& Rengo, G. (2019). Periodontal disease: A risk factor for diabetes and cardiovascular disease. International Journal of Molecular Sciences, 20(6), 1-14.

Lindemark, F., Haaland, Ø. A., Kvåle, R., Flaatten, H., \& Johansson, K. A. (2015). Age, risk, and life expectancy in Norwegian Intensive Care: A registrybased population modelling study. PLOS ONE, 10(5), 1-16.

Macedo, F. R., Saba-Chujfi, E., Pereira, S. A. S., Costa, E. L. da, \& Melo Neto, J. P. de. (2010). Association between periodontitis and lung disease. RGO (Porto Alegre), 47-53.

Meidani, M., Khorvash, F., Abbasi, S., Cheshmavar, M., \& Tavakoli, H. (2018). Alloimmunization in thalassemia patients: New insight for healthcare. International Journal of Preventive Medicine, 8, 2018-2021.

Munro, C. L., \& Grap, M. J. (2004). Oral health and care in the intensive care unit: State of the science. American Journal Of Critical Care, $13(1)$, 25-35.

Raber-Durlacher, J. E., Weijl, N. I., Saris, M. A., Koning, B. de, Zwinderman, A. H., \& Osanto, S. (2000). Oral mucositis in patients treated with chemotherapy for solid. Supportive Care in Cancer, 8(5), 366-371.

Ridker, P. M., Buring, J. E., Shih, J., Matias, M., \& Hennekens, C. H. (1998). Prospective study of C-reactive protein and the risk of future cardiovascular events among apparently healthy women. Circulation, 98(8), 731-733.

Silva, J. L., El Kadre, G. D. A. de O., Kudo, G. A. H., Junior, J. F. S., \& Saraiva, P. P. (2016). Oral health of patients hospitalized in the intensive care unit. Journal of Contemporary Dental Practice, 17(2), 125-129.

Sanz, M., del Castillo, A. M., Jepsen, S., Gonzalez-Juanatey, J. R., D’Aiuto, F., Bouchard, P., Chapple, I., Dietrich, T., Gotsman, I., Graziani, F., Herrera, D., Loos, B., Madianos, P., Michel, J. B., Perel, P., Pieske, B., Shapira, L., Shechter, M., Tonetti, M., \& Wimmer, G. (2020). Periodontitis and cardiovascular diseases: Consensus report. Journal of Clinical Periodontology, 47(3), 268-288.

Scannapieco, F. A. (2006). Pneumonia in nonambulatory patients: The role of oral bacteria and oral hygiene. Journal of the American Dental Association, 137(10 SUPPL.), 21-25.

Scannapieco, F. A., \& Rethman, M. P. (2003). The relationship between periodontal diseases and respiratory diseases. Dent Today, 22 , 79-83.

Silva, C. B. C. da. (2019). Perfil de pacientes internados em unidade de terapia intensiva e fatores associados ao desenvolvimento de mucosite oral. In Universidade Federal do Rio Grande do Norte. 
Research, Society and Development, v. 10, n. 4, e58910414444, 2021

(CC BY 4.0) | ISSN 2525-3409 | DOI: http://dx.doi.org/10.33448/rsd-v10i4.14444

Sprung, C. L., Danis, M., Iapichino, G., Artigas, A., Kesecioglu, J., Moreno, R., Lippert, A., Curtis, J. R., Meale, P., Cohen, S. L., Levy, M. M., \& Truog, R. D. (2013). Triage of intensive care patients: Identifying agreement and controversy. Intensive Care Medicine, 39(11), 1916-1924.

Tulio, K. de S. C., Stramandinoli-Zanicotti, R. T., Dirschnabel, A. J., Schussel, J. L., Wasilewski, J. H. S., Krelling, A., Beltrame, O. C., Martins, C. R. W., \& Sassi, L. M. (2018). Alterações no perfil da microbiota bucal durante permanência na UTI : colonização por patógenos respiratórios potenciais Pacientes sob cuidados intensivos constituem um grupo de indivíduos em condições graves , A Unidade de Terapia Intensiva ( UTI ) repre. 7, 351-357.

Vidal, C. F. D. L. (2014). Impacto da Higiene Oral com clorexidina com e sem escovação dental na Prevenção da Pneumonia associada à Ventilação Mecânica: Estudo Randomizado Impacto da Higiene Oral com clorexidina com e sem escovação dental na Prevenção da Pneumonia as sociada à Vent. https://repositorio.ufpe.br/bitstream/123456789/12053/1/TESE Claudia Fernanda de Lacerda Vidal.pdf\%3E

Wunsch, H., Angus, D. C., Harrison, D. A., Linde-Zwirble, W. T., \& Rowan, K. M. (2011). Comparison of medical admissions to intensive care units in the United States and United Kingdom. American Journal of Respiratory and Critical Care Medicine, 183(12), 1666-1673. 